\title{
Audit of Diabetes Knowledge among Adults with Diabetes in Bobo-Dioulasso (Burkina Faso): A Cross-Sectional Study Prior to Intervention Strategy
}

\author{
Téné Marceline Yaméogo 1,2*, Carole Gilberte Kyelem,2, Oumar Guira33, Issiaka Sombié1, \\ Djingri Lankoandé2, Annick Raïssa Ouelhôre Sidibé ${ }^{1}$, Alassane Ilboudo², \\ Aimée Sankaye-Lagom Kissou 1,2, Abraham Bagbila², Apollinaire Sawadogo 2,3, \\ Macaire Sampawinde Ouédraogo ${ }^{1,2}$, Youssouf Joseph Drabo ${ }^{3}$
}

${ }^{1}$ INSSA, University Nazi Boni de Bobo-Dioulasso, Bobo-Dioulasso, Burkina Faso

${ }^{2} \mathrm{CHU}$ Sourô Sanou de Bobo-Dioulasso, Bobo-Dioulasso, Burkina Faso

${ }^{3}$ UFR-SDS, University Ouaga I Joseph Ki Zerbo, Ouagadougou, Burkina Faso

Email: ${ }^{\star}$ teneline@gmail.com

How to cite this paper: Yaméogo, T.M., Kyelem, C.G., Guira, O., Sombié, I., Lankoandé, D., Sidibé, A.R.O., Ilboudo, A., Kissou, A.S.-L., Bagbila, A., Sawadogo, A., Ouédraogo, M.S. and Drabo, Y.J. (2018) Audit of Diabetes Knowledge among Adults with Diabetes in Bobo-Dioulasso (Burkina Faso): A Cross-Sectional Study Prior to Intervention Strategy. Open Journal of Internal Medicine, 8, 10-17. https://doi.org/10.4236/ojim.2018.81002

Received: December 10, 2017

Accepted: January 19, 2018

Published: January 22, 2018

Copyright $\odot 2018$ by authors and Scientific Research Publishing Inc. This work is licensed under the Creative Commons Attribution International License (CC BY 4.0).

http://creativecommons.org/licenses/by/4.0/

\begin{abstract}
Several studies have shown the importance of therapeutic education in the management of diabetes. Its implementation requires a prior assessment of the level of patients' knowledge. The objective of this study was to assess the level of diabetics' knowledge about their disease. Methods: This was a cross-sectional study conducted between 2013 and 2015; consenting diabetics, followed for at least one year in medicine department of Souro Sanou Hospital, have been surveyed. The data were collected using a 51-item questionnaire, adapted from "Audit of Diabetes Knowledge" and administered by an interviewer. The level of knowledge was satisfactory/good if at least $80 \%$ of the set items or a topic were known. Results: A total of 268 diabetics were surveyed. Their mean age was $54.3 \pm 1.4$ years and the sex ratio 0.8 . The majority (80.9\%) lived in urban areas and half (50.4\%) were uneducated. Type 2 diabetics accounted for $93.3 \%$ of the population; the average seniority was $6.0 \pm$ 0.7 years. The level of knowledge was generally insufficient, with an average total score of $25.4 \pm 1.0 / 51$. The topics which were not well mastered related to diet $(0.8 \%)$, glycated haemoglobin $(4.5 \%)$, and signs of hypoglycemia (7.8\%). The main relatively well-known topics related to the effect of physical exercise on glycemia $=37.7 \%$, the effect of food on glycemia $=36.9 \%$ and glycemia target $=32.8 \%$. Conclusion: This study revealed a lack of knowledge from diabetic patients who are followed in the medicine department of the Teaching Hospital Souro SANON (CHUSS). The implementation of a therapeutic education program for diabetics is necessary and urgent.
\end{abstract}




\section{Keywords}

Diabetes, Knowledge, Education, Burkina

\section{Introduction}

The growing prevalence of diabetes raises the problem of its prevention and its adequate management. Several studies have shown the importance of therapeutic education in understanding the disease, the adhesion to therapeutic plans and glycemia control [1] [2] [3] [4].

Several studies have demonstrated a poor disease knowledge among people with diabetes in developed countries. For example, in Speight and Bradley's study in United Kingdom, 57\% cold not recognize the inaccuracy of the statement: "Fresh fruit can be eaten freely with little effect on blood glucose levels". $75 \%$ of patients did not know that it is advisable to trim toenails to the shape of the toe [5]. In Poulimeneas' study in Greece, the mean score of Diabetes Knowledge Test was $8.3 \pm 2.2 / 14.0$ [6].

In a context of limited resources like ours, various constraints hinder the adequate management of diabetics. In the Teaching Hospital of the city, the care of diabetics was done in a pavilion intended for general intern and ambulatory medicine (outpatient) by an endocrinologist and three intern doctors, helped by a polyvalent medical and paramedical staff. An evaluation of these patients in 2011-2 showed that only 37\% had a satisfactory knowledge level on general questions about diabetes and that this knowledge level was decisive in their adhesion to the follow-up and implementation of annual paraclinical prescriptions [7]. This study was conducted with the aim of having a thorough educational diagnosis with a view to developing an action plan to improve the supply of therapeutic education.

\section{Methods}

This was a cross-sectional study conducted between 2013 and 2015 among diabetics admitted to consultation and/or hospitalized in the department of medicine. It was an exhaustive sampling; all diabetic patients meeting inclusion criteria and who were received during the study period were recruited. Were consecutively included, all diabetics aged at least 17 , who were consenting, who were followed for at least one year in the department and who speak French, Dioula and Mooré (the 2 main local languages of the city). Diabetics who met inclusion criteria but who were physically unable to answer the questionnaire (aphasic, comatose) were excluded.

The survey questionnaire was adapted from C. Bradley's Audit of Diabetes Knowledge (ADKnowl), (Royal Holloyay University London, UK) [8]. It was composed of 51 questions under 8 topics: General knowledge with 18 questions, treatment with 6 questions, signs of hypoglycemia with 6 questions, treatment of 
hypoglycemia with 3 questions, effects of physical exercise with 3 questions, diet/effect of food on glycemia with 8 questions, diabetic foot with 3 questions, glycemia equilibrium with 4 questions. The questionnaire was administered by interview by interviewers (nurses) trained for that and through an individual collection form.

Each correct answer was scored 1. The level was considered satisfactory/good for all the questions when at least $80 \%$ of answers were correct (for example thescore $\geq 41 / 51$ for all the questions), average if correct answers were between $50 \%$ and $80 \%$ (for example thescore $\geq 26$ to $40 / 51$ ), and insufficient when correct answers were less than 50\% (for example thescore $\leq 25 / 51$ ).

For each topic, the level was considered satisfactory/good when at least $80 \%$ of answers were correct and insufficient when correct answers were less than $80 \%$. For example, the signs of hypoglycemia knowledge were assessed through 6 questions; the level for this topic was considered satisfactory/good when the score was $\geq 5 / 6$ and insufficient when the score was $<5 / 6$. The average of the scores of a topic is the total of individual scores divided by the number of diabetics surveyed.

A statistical analysis was performed using Stata 12. Pearson and Student's Chi2 tests were used at $5 \%$ significance level.

The study obtained the approval of the national ethics committee $\left(\mathrm{N}^{\circ}\right.$ 2013-6-47) and that of Teaching Hospital officials. An information leaflet and a consent form for the survey were provided to each patient surveyed. A study was conducted with respect for the anonymity and confidentiality of respondents.

\section{Results}

A total of 268 diabetics were included. Their mean age was $54.3 \pm 1.4$ years and the sex ratio 0.8 . The majority, $86.8 \%$, lived in urban areas, that is an urban/rural ratio of $7: 1 ; 50.4 \%$ were out of school and $56.7 \%(n=152)$ had a regular income.

Type 2 diabetics accounted for $93.3 \%$ of the population. The average seniority since the discovery of diabetes was $6.0 \pm 0.7$ years; $55.5 \%(n=148)$, had their diabetes discovered for less than 5 years.

\subsection{Overall Knowledge Score on Diabetes}

The overall mean score was $25.4 \pm 1.0 / 51$, with extremes of 1 and $47 / 51$.

Only two (02) diabetics, that is $0.7 \%$, had a good knowledge level; for $49.0 \%$ it was average. The overall mean score of out-of-school and primary-school diabetics $(\mathrm{n}=210)$ was $29.1 / 51$ against $24.3 / 51$ for those who had secondary and higher education level $(n=58)$; the difference was statistically significant $(\mathrm{p}=$ $0.0002)$.

There was no difference by age, gender, seniority or type of diabetes or place of residence (urban/rural).

\subsection{General Knowledge about Diabetes}

The mean score was $10.4 \pm 0.4 / 18$, with extremes of 0 and $17 / 18$. The level was 
good for $8.2 \%$ of diabetics $(\mathrm{n}=22)$, average for $67.2 \%(\mathrm{n}=180)$ and insufficient for $24.3 \%(n=66)$.

\subsection{Specific Knowledge of Topics}

The general level was average for each of the topics assessed, except for diet and glycemia target for which the mean score was bad: $0.5 \pm 0.1 / 3$ and $0.3 \pm 0.3 / 1$, respectively (Table 1 ).

Apart from diet and glycemia target topics, the diabetics surveyed had approximatelyan average knowledge of the topics covered.

The main relatively well-known topics by diabetics (about one-third) related to the effect of physical exercise on glycemia $=37.7 \%$, the effect of food on glycemia $=36.9 \%$, glycemia target $=32.8 \%$ (Figure 1 ). Only $17.5 \%$ had a good knowledge level on diabetic foot care.

Table 1. Mean and extremes scores on topics assessed about diabetes knowledge.

\begin{tabular}{ccc}
\hline Topic & Mean score $\mathrm{N}=268$ & Extremes score \\
\hline Overall knowledge/51 & $25.4 \pm 1.0$ & $1-47$ \\
General knowledge/18 & $10.4 \pm 0.4$ & $0-18$ \\
Treatment/6 & $2.8 \pm 0.2$ & $1-6$ \\
Signs of hypoglycemia/6 & $2.8 \pm 0.2$ & $1-3$ \\
Treatment of hypoglycemia/3 & $1.7 \pm 0.2$ & $1-3$ \\
Effect of physical exercice/3 & $1.7 \pm 0.2$ & $1-3$ \\
Diet/3 & $0.5 \pm 0.1$ & $0-5$ \\
Effect of food on glycemia/5 & $2.8 \pm 0.2$ & $1-3$ \\
Diabetic foot care/3 & $1.5 \pm 0.9$ & $0-1$ \\
Glycemia target/1 & $0.3 \pm 0.3$ & $1-3$ \\
Glycated haemoglobin/3 & $0.3 \pm 0.1$ & 1 \\
\hline
\end{tabular}

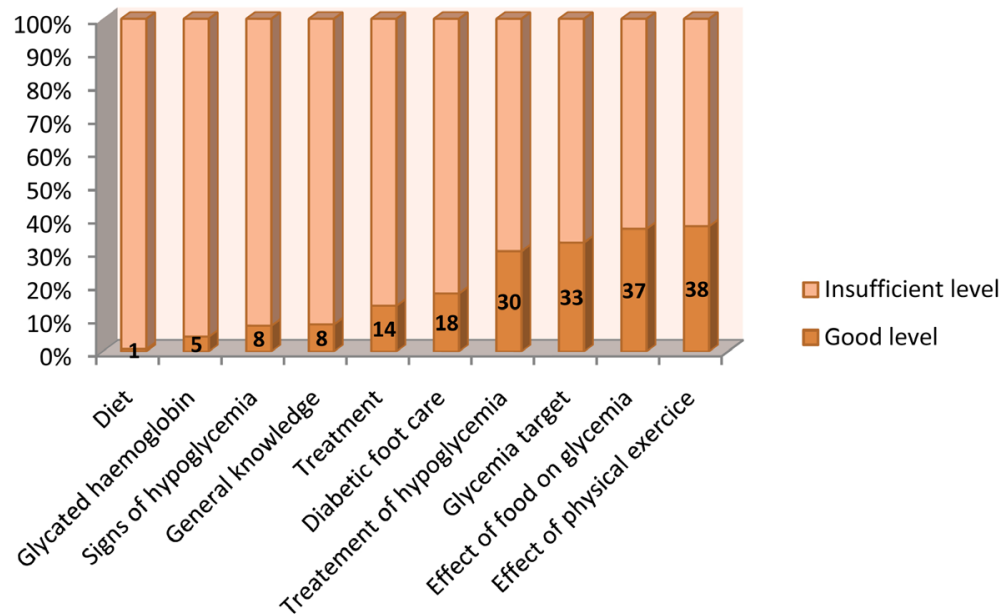

Figure 1. Specific knowledge of topics on diabetes among people suffering from diabetes in Bobo-Dioulasso. 
The least known topics concerned diet $=0.8 \%$, glycated haemoglobin target $=$ $4.5 \%$, signs of hypoglycemia $=7.8 \%$ (Figure 1 ).

\section{Discussion}

The level of knowledge of diabetics was generally insufficient.

Many studies show that patient adhesion to care project, one of the essential elements for a good management of chronic diseases, is linked to the knowledge of the disease [1] [2] [3] [4] [7]. In order to adhere to treatment, it is necessary that the patient has the control of his illness, from the diagnosis to the treatment through complications and especially that he knows and understands the importance of the follow-up. Yet, therapeutic education is still insufficient in our countries. In Bobo-Dioulasso, there is no center dedicated to the care of patients. As far as diabetes is concerned, education is only provided during doctor's consultations, which is far insufficient.

This study is a review of the knowledge of our patients, more systematized than the preliminary study of 2011 [7]. It reveals a general level of knowledge about diabetes which is insufficient; only 2 out of 268 patients had a good overall knowledge of the disease. The overall mean score was $25.4 \pm 1.0 / 51$.

The conclusions of the studies are variable on the issue and are difficult to compare because of methodological differences. However, in general, the level of knowledge of diabetics is considered insufficient [6] [9] [10] [11].

In France, a poor level of knowledge about diabetes was found by Roossens among type 2 diabetics treated only by ADO [10]. Al-Adsani in Kuwait also found the same results with his population of type 2 diabetics. Although he found a mean score of $58.9 \%$ of correct answers, only $9.7 \%$ of patients had a good knowledge level [11]. However, Moodley in South Africa found a satisfactory level of knowledge in $66.9 \%$ of cases [12].

In our context many factors may explain the weakness of the overall level of patient knowledge.

- Lack of qualified staff: In Burkina Faso, there is less than 1 (0.45) physician per 10,000 people, 3.57 nurses per 10,000 people [13]. Diabetes monitoring at Teaching Hospital Souro SANON (CHUSS) is provided by only four specialist doctors, namely three internists and one endocrinologist, to whom all patients from the western half of the country are referred. Apart from the internists and the endocrinologist, diabetics are also cared for in hospital by nurses who care for patients suffering from various other pathologies.

- Low knowledge level of health workers: Sawadogo in 2013 in a study on the level of knowledge of health workers in the city of Bobo on diabetes showed an insufficient level in $93.3 \%$ of surveyed health workers (in press). The reasons given were inadequate initial training of health professionals on diabetes; the absence of a permanent diabetes education program, particularly at Teaching Hospital Souro SANON (CHUSS); the absence of a national program to combat diabetes or cardiovascular diseases in general. 
- Insufficiency of ETP activities: the lack of a unit dedicated to the management of diabetes at Teaching Hospital Souro SANON (CHUSS) allowing extensive educational activities represents a barrier to educational activities. As a matter of fact, ETP in our context is only performed during the patient follow-up consultation. Although medical specialists in the field strive as much as possible to ensure the education of patients at each consultation, the workload does not always allow them to give patients the necessary time. In addition, to be effective over the long term, educational activities should not be limited to individual interventions. As a matter of fact, the impact of group education in addition to individual interventions is important and should justify the implementation of this type of intervention.

- Low educational level: We noted an association between the knowledge of the disease and the level of education. This observation was also made by $\mathrm{Al}$-adsani in Kuwait [11], and Moodley in South Africa [12]. The poor level of education in Burkina Faso in general may, therefore, partly explain the low level of knowledge about diabetes.

Diabetic foot is the leading cause of non-traumatic limb amputation in developed countries where its frequency is estimated at between 5\% and 15\% [14]. A good knowledge level on the diabetic foot was found in $17.5 \%$ of followed patients. This is very insufficient when we know that it is a frequent complication that can lead to amputation and whose prevention is easy through therapeutic education.

Two points explain the shortcomings of the study:

- The ADKnowl that inspired our questionnaire was translated from English to French. The services of a translator were used in order to minimize misunderstandings. Similarly, the questionnaire was submitted to several reviews within the project team and to a pre-test to diabetics before being validated.

- The translation of questions from French into national languages Dioula and Mooré for their administration to diabetics who do not understand French could also be the cause of comprehension errors. In order to minimize this possibility, the 5 interviewers were trained on the questionnaire, question by question, in order to clarify their meaning and their best formulation in local languages. Diabetics who did not understand these 3 languages were not included in the study.

\section{Conclusion}

This study has permitted to assess the level of knowledge of diabetics followed in the CHUSS medicine department between 2013 and 2015 on their disease. It made it possible to underline notorious insufficiencies in their knowledge. None of the 8 topics assessed were sufficiently mastered and the most alarming is that dietary measures, essential element of the treatment, as well as the indicators of the glycemia equilibrium, were the least mastered topics. It is urgent to set up at Teaching Hospital Souro SANON (CHUSS) an unremitting program of educa- 
tion for diabetics.

\section{Acknowledgements}

WAEMU (UEMOA) Support Program for Higher Education.

\section{References}

[1] Al Hayek, A.A., Robert, A.A., Al Dawish, M.A., Zamzami, M.M., Sam, A.E. and Alzaid, A.A. (2013) Impact of an Education Program on Patient Anxiety, Depression, Glycemic Control, and Adherence to Self-Care and Medication in Type 2 Diabetes. Journal of Family and Community Medicine, 20, 77-82. https://doi.org/10.4103/2230-8229.114766

[2] Malathy, R., Narmadha, M., Ramesh, S., Alvin, J.M. and Dinesh, B.N. (2011) Effect of a Diabetes Counseling Program on Knowledge, Attitude and Practice among Diabetic Patients in Erode District of South India. Journal of Young Pharmacists, 3, 65-72. https://doi.org/10.4103/0975-1483.76422

[3] Cave, C., Green, C., Royle, P., Dunn, N. and Waugh, N. (2003) The Clinical and Cost-Effectiveness of Patient Education Models for Diabetes: A Systematic Review and Economic Evaluation. Health Technology Assessment, 7, 1-190.

[4] Oosthuizen, H., Riedijk, R., Nonner, J., Rheeder, P. and Ker, J.A. (2002) An Educational Intervention to Improve the Quality of Care of Diabetic Patients. South African Medical Journal, 92, 459-464.

[5] Speight, J. and Bradley, C. (2001) The ADKnowl: Identifying Knowledge Deficits in Diabetes Care. Diabetic Medicine, 18, 626-633. https://doi.org/10.1046/j.1464-5491.2001.00537.x

[6] Poulimeneas, D., Grammatikopoulou, M.G., Bougioukli, V., Iosifidou, P., Vasiloglou, M.F. and Gerama, M.A. (2016) Diabetes Knowledge among Greek Type 2 Diabetes Mellitus Patients. Endocrinología y Nutrición, 63, 320-326.

[7] Yaméogo, T.M., Rouamba, N., Sombié, I., Djingri, L., Kyelem, C.G., Ouédraogo, M.S., et al. (2016) Contributing Factors to Diabetics' Compliance for Their Follow-Up in Resource-Limited Settings (Burkina Faso). RAFMI, 3, 17-22.

[8] Bradley. ADKnowl/Audit of Diabetes Knowledge. https://www.healthpsychologyresearch.com/guidelines/adknowl-audit-diabetes-kno wledge

[9] Mukeshimana, M.M. and Nkosi, Z.Z. (2014) Communities' Knowledge and Perceptions of Type Two Diabetes Mellitus in Rwanda: A Questionnaire Survey. Journal of Clinical Nursing, 23, 541-549. https://doi.org/10.1111/jocn.12199

[10] Roossens, P.J., Poulalion, L., Beigbeder, I., Fesquet, E. and Becel, B. (2000) Identification of Factors Hindering Better Management of Patients with Type 2 Diabetes. Diabetes \& Metabolism, 26, 77-85.

[11] Al-adsani, A.M.S., Moussa, M.A.A. and Al-jasem, L.I. (2009) The Level and Determinants of Diabetes Knowledge in Kuwaiti Adults with Type 2 Diabetes. Diabetes \& Metabolism, 35, 121-128. https://doi.org/10.1016/j.diabet.2008.09.005

[12] Moodley, L. and Rambiritch, V. (2007) An Assessment of the Level of Knowledge about Diabetes Mellitus among Diabetic Patients in a Primary Healthcare Setting. South African Family Practice, 49, 16. https://doi.org/10.1080/20786204.2007.10873652

[13] WHO, Global Health Workforce Alliance (2017) Burkina Faso. 
http://www.who.int/workforcealliance/countries/bfa/en/

[14] Lamchahab, F., El Kihal, N., Khoudri, I., Chraibi, A., Hassam, B. and Ourhroui, M. (2011) Factors Influencing the Awareness of Diabetic Foot Risks. Annals of Physical and Rehabilitation Medicine, 54, 359-365.

https://doi.org/10.1016/j.rehab.2011.07.004 